\title{
Patterns of Relating Between Physicians and Medical Assistants in Small Family Medicine Offices
}

\author{
Nancy C. Elder, MD, MSPH ${ }^{1}$ \\ C. Jeffrey Jacobson, $P b D^{1,2}$ \\ Sbannon K. Bolon, MD, MPH \\ Joseph Fixler, BA ${ }^{4}$ \\ Harini Pallerla, $M S^{1}$ \\ Christina Busick, $B A, B S^{1}$ \\ Erica Gerrety, BSN ${ }^{5}$ \\ Dee Kinney, MA, RD, $C M A^{6}$ \\ Saundra Regan, $P b D^{1}$ \\ Michael Pugnale, BS
}

'University of Cincinnati Department of Family and Community Medicine,

Cincinnati, Ohio

${ }^{2}$ University of Cincinnati Department of Anthropology, Cincinnati, Ohio

${ }^{3}$ Bureau of Health Professions, Health Resources and Services Administration, Cincinnati, Ohio

${ }^{4}$ University of Cincinnati College of Medicine, Cincinnati, Ohio

${ }^{5}$ University of Cincinnati College of Nursing, Cincinnati, Ohio

${ }^{6}$ University of Cincinnati Clermont College, Cincinnati, Ohio

Conflicts of interest: authors report none.

\section{CORRESPONDING AUTHOR}

Nancy C. Elder, MD, MSPH

Department of Family and Community Medicine

University of Cincinnati

PO Box 670582

Cincinnati, $\mathrm{OH}$ 45267-0582

Nancy.elder@uc.edu

\begin{abstract}
PURPOSE The clinician-colleague relationship is a cornerstone of relationship-centered care (RCC); in small family medicine offices, the clinician-medical assistant (MA) relationship is especially important. We sought to better understand the relationship between MA roles and the clinician-MA relationship within the RCC framework.
\end{abstract}

METHODS We conducted an ethnographic study of 5 small family medicine offices (having $<5$ clinicians) in the Cincinnati Area Research and Improvement Group (CARInG) Network using interviews, surveys, and observations. We interviewed 19 MAs and supervisors and 11 clinicians (9 family physicians and 2 nurse practitioners) and observed 15 MAs in practice. Qualitative analysis used the editing style.

RESULTS MAs' roles in small family medicine offices were determined by MA career motivations and clinician-MA relationships. MA career motivations comprised interest in health care, easy training/workload, and customer service orientation. Clinician-MA relationships were influenced by how MAs and clinicians respond to their perceptions of MA clinical competence (illustrated predominantly by comparing MAs with nurses) and organizational structure. We propose a model, trust and verify, to describe the structure of the clinician-MA relationship. This model is informed by clinicians' roles in hiring and managing MAs and the social familiarity of MAs and clinicians. Within the RCC framework, these findings can be seen as previously undefined constraints and freedoms in what is known as the Complex Responsive Process of Relating between clinicians and MAs.

CONCLUSIONS Improved understanding of clinician-MA relationships will allow a better appreciation of how clinicians and MAs function in family medicine teams. Our findings may assist small offices undergoing practice transformation and guide future research to improve the education, training, and use of MAs in the family medicine setting.

Ann Fam Med 2014;150-157. doi:10.1370/afm.1581.

\section{INTRODUCTION}

M edical assistants (MAs) have become the primary clinical assistant in family medicine offices, replacing registered and licensed practical nurses. ${ }^{1,2}$ Laws governing MA practice vary by state ${ }_{i}$ but these professionals most commonly work under the license of a supervising physician, not under MA-specific laws. ${ }^{3,4} \mathrm{MA}$ training is highly variable, ranging from entirely on the job to 2-year college degrees; however, 2 voluntary certification bodies exist. ${ }^{2}$

A 2006 systematic literature review on the roles of MAs identified 18 articles, all predating 1997; 13 were published before 1989, providing more historical context than insight into how MAs function today. ${ }^{2}$ Chapman et $\mathrm{al}^{5,6}$ and Taché and Hill-Sakurai ${ }^{7}$ recently interviewed MAs, physicians, and MA educators in California. They found a consensus that MAs' proficiencies lie in patient flow and continuity, and that their role is to facilitate the patient visit. ${ }^{5,7}$ In small family medicine offices, however, the entire team is often limited to physicians and MAs, giving the latter increasingly 
diverse roles and making their relationship central to office function. ${ }^{6,8}$ Most MAs are not trained for such innovative roles, however. ${ }^{5}$

The need for office teams continues to grow, ${ }_{1}^{9-11}$ as does the essential role of teamwork in practice transformation, yet family medicine team models remain relatively underdeveloped..$^{12}$ The clinician-colleague relationship is a cornerstone of relationship-centered care (RCC). Unlike the case of clinician-patient relationships, however, qualities of clinician-colleague relationships remain relatively unstudied ${ }_{i}$ most existing research examines hospital-based physicians and nurses. ${ }^{13}$

Understanding relationship qualities and patterns of relating (or teamwork) within health care settings requires knowledge of professional and institutional cultures and hierarchies. ${ }^{14,15}$ These patterns are nonlinear and self-organizing, and are subject to both freedoms and constraints. A complexity-inspired theory of human interaction called Complex Responsive Processes of Relating (CRPR) provides a theoretical framework for viewing such relational dynamics in organizations. ${ }^{15}$ Within small family medicine offices, specific constraints and degrees of freedom shaping the clinician-MA relationship have not been fully delineated..$^{15}$ We intensively studied small family medicine offices through interviews, surveys, and observations to better understand MA roles and describe the clinician-MA relationship within an RCC organizational model of family medicine. ${ }^{13-15}$

\section{METHODS}

\section{Participants}

We invited 5 small family medicine offices (having $<5$ clinicians) in the Cincinnati Area Research and Improvement Group (CARInG) practice-based research network to participate. CARInG is a regional primary care network consisting of 21 family and general internal medicine offices. We sought offices with differences in factors that might affect how MAs function, including setting, clinician characteristics, practice age, and ownership. Although MA characteristics were considered important, these were not available until after an office joined the study. Within each office, we invited all MAs and clinicians (family physicians and nurse practitioners) to participate. Four offices had an MA clinical supervisor, while at the fifth, the head clinician supervised the staff. "Supervisor" MAs also performed the clinical work of other MAs; therefore, supervisor and MA data were aggregated as appropriate. This study was approved by the University of Cincinnati Institutional Review Board and was performed between July 2010 and March 2011.

\section{Data Collection}

We developed survey questionnaires and interview guides based on the literature $\mathrm{e}^{1,2,4,8}$ and on informal interviews and pilot testing with nonparticipant MAs, clinicians, and an MA educator. We collected office and individual demographics, training information, and work experiences using the questionnaires. Participants completed a checklist of tasks performed by MAs and indicated whether they, or MAs at their practice, performed each task.

Three experienced qualitative researchers conducted semistructured, audio-recorded interviews with clinicians and MAs. Clinicians were asked how MAs came to be the clinical assistants in their office, how they interacted with MAs, and what their experiences were with changing MA roles and activities. MAs were asked about how and why they became an MA, their daily work activities, and their interactions and communications with clinicians. The same research team members performed direct continuous observations of the MAs. Every 5 minutes or when a behavior or activity changed, observers noted MAs' activities and location (eg, examination room, hallway).

\section{Data Analysis}

We entered quantitative demographics and survey responses into a database, and calculated descriptive frequencies with IBM SPSS 19.0 (SPSS Inc). Qualitative data (interview transcripts and observation notes) were entered into NVivo 8.0 (QSR International) for management and analysis. All transcripts and notes were initially coded into categories related to the interview questions and then refined by revisiting the transcripts, consistent with the editing style, to characterize teams and teamwork according to the CRPR and RCC frameworks. ${ }^{16,17}$ We then developed case studies ${ }^{18}$ and created a model of MA roles based on how role-related experiences and conditions directed and constrained clinician-MA team self-organization. Approximately 18 months after original data collection, and outside the study, we obtained each office's National Committee for Quality Assurance (NCQA) Patient-Centered Medical Home (PCMH) certification information through publicly available sources.

\section{RESULTS}

Following the CRPR framework, we first consider role expectations and perceptions and then describe key components of a clinician-MA relationship model. Case studies of the 5 offices demonstrate how different relational patterns are affected by constraints and degrees of freedom defined by the model (Table 1).

Only a single MA declined to be interviewed, yielding 19 MAs and 11 clinicians (9 family physi- 
cians and 2 nurse practitioners) (Table 2). Fifteen MAs were observed for a total of 100.5 hours (range $=2-11$ each, mean $=6.5$, median $=7$ hours). We observed 2 to 4 MAs at each office, for a mean of 20.1 observation hours per office (median $=22$, range $=11.5-23.5$ hours) . Staff meetings were observed at 4 offices. Engagement with each office depended on MA and clinician availability and ranged from 1 to 4 weeks.

\section{Role Expectations and Perceptions}

We identified 2 factors that provide background for understanding clinician-MA relationships in smaller

\section{Table 1. Case Studies of Clinician-MA Relationships}

\section{Office 1: Mature practice, physician couple owned}

At this decade-old, 2-physician-owned office, the physicians personally hired all the MAs. Physicians and a senior MA who functions as a supervisor shared daily management. There was little socializing, but there were regular meetings held between the physicians and the MAs. No regular evaluations of the MAs were performed by the physician or the supervisor. There was low MA turnover. The physicians did not allow the MAs to answer patient questions without physician permission, but the MAs stated they performed this function independently. Follow-up: office has not applied for NCQA PCMH certification.

\section{Office 2: Mature practice, formerly physician owned, now health system owned}

This decade-old, 3-clinician (2-physician, 1-FNP) office was recently purchased from the physician owners by a nonprofit health system. The physicians initially hired the MAs, who were managed by the office manager; however, responsibility for these tasks was less clear under the new ownership. Frequent socializing occurred between clinicians and MAs within the office setting, and regular meetings were held between the staff and clinicians. The office manager has performed evaluations of the MAs with clinician input in the past, but not recently. There was low MA turnover. The clinicians felt they had adequately trained the MAs over the years and trusted their medical acumen to answer patient questions and provide patient education. Follow-up: office has NCQA PCMH certification.

\section{Office 3: Mature practice, physician owner retired, now health system owned}

This decades-old solo physician office was recently purchased by a nonprofit health system when the owner retired. A relatively new physician was hired for the office. The MAs included those who worked with the previous physician and new hires by the health system. The physician socialized and ate lunch with the MAs and held regular meetings with the staff. Neither the physician nor the MA supervisor performed regular evaluations of the MAs. There was MA turnover during the sale of the practice, but little since then. The clinician felt she knew the MAs from their meetings and frequent conversations and trusted their medical acumen to answer patient questions and provide patient education. Follow-up: office has NCQA PCMH certification.

\section{Office 4: New practice, health system owned}

This 2-year-old, 3-physician office was started by a nonprofit health system to build a practice that in the future might house a family medicine residency. The physicians were recent graduates. All the MAs were hired by the health system with no physician input. No physician had responsibility for training or overseeing the MAs; the MA supervisor and office manager for several primary care offices provided direct management to the MAs. There was minimal socializing at lunch or breaks between MAs and clinicians. The physicians and staff held regular meetings. There was no regular evaluation or feedback of MA performance. There was high MA turnover. The clinicians did not trust their MAs' clinical acumen, but they did not train or oversee them. Follow up: major physician and staff personnel changes, office has NCQA PCMH certification.

\section{Office 5: Mature practice, physician owned}

At this decades-old, 2-clinician (physician, nurse practitioner), physician-owned office, the physician personally hired all the MAs and provided day-to-day management. MAs and clinicians frequently lunched on site together, there were regular meetings between staff and clinicians, and each year, the practice closed for several days and the physician took all the staff on a short vacation together. Although there was some business conducted, it was primarily a social event. The physician clearly defined both the clinical and clerical roles, but regular evaluation and feedback was inconsistent. Communication between MAs and between MAs and clinicians occasionally escalated to tears. There was little staff turnover. There were strict protocols for what clinical advice MAs could give to patients, and work was in a centra area where physician was able to observe patient-MA interactions. Follow-up: office has not applied for NCQA PCMH certification.

FNP = family nurse practitioner; $M A=$ medical assistant; $N C Q A=$ National Committee for Quality Assurance; $\mathrm{PCMH}=$ patient-centered medical home. family medicine offices: MAs' career motives and dis-

\section{MAs' Career Motives}

MAs described 3 main categories of motives for choosing medical assisting: interest or experience in health care, easy training and workload, and desire to work with people. Among the 14 for whom becoming an MA was a second career, 6 had worked in a different medical career (emergency medical technician, nurse assistant, or hospital clerk), 2 had some nursing not licensed nurses), and 3 had performed medical office work; however, most had held many past jobs, including nonmedical office, retail, and manufacturing jobs.

The ease of MA training, regular hours, and minimal physical requirements were key factors mentioned by 13 participants. Several MAs learned entirely on the job; others found that brief and focused training better fit their needs; as one MA remarked, "I just had no interest in taking classes that were pointless for a health field....The school that I went to is more of a technical class, so all my classes were strictly what I needed for medical assisting." A few noted they could no longer do heavy lifting or needed regular 9-to-5 hours.

Nine MAs described a desire to work with people. One MA's comment, "I'm such a people person, I need to be where the patients are, and deal with the patients one on one," expressed this motive, while also introducing the situation of MAs dealing "with the patients one on one," in which we found potential conflict between MAs and clinicians.

\section{Disagreement About MAs' Clinical Competence}

In interviews, MAs and clinicians agreed that MA's primary responsibility is maintaining patient flow by checking in patients (reported by 18 of 19 MAs and 9 of 11 clinicians), preparing examination rooms (15 of 19 MAs, 9 of 
11 clinicians), and directly assisting the clinician (18 of 19 MAs, 9 of 11 clinicians). Our observations corroborated these views, but also found MAs commonly performed clerical duties such as handling patient telephone calls, filing, faxing, and maintaining equipment.

Table 2. Characteristics of Participating Clinicians, MAs, and Offices

\begin{tabular}{|c|c|c|c|c|c|}
\hline Characteristic & Office 1 & Office 2 & Office 3 & Office 4 & Office 5 \\
\hline \multicolumn{6}{|l|}{ Clinicians $^{a}$} \\
\hline Number & 2 & 3 & 1 & 3 & 2 \\
\hline Sex, \% women & 50 & 33 & 100 & 33 & 100 \\
\hline Age, mean, y & 44 & 51 & 30 & 34 & 55 \\
\hline Race, \% white & 0 & 66 & 100 & 100 & 100 \\
\hline Mean years as clinician & 13 & 27 & 2 & 7 & 13 \\
\hline Mean years at this office & 12 & 19 & 1 & 2 & 9 \\
\hline \multicolumn{6}{|l|}{ MAs } \\
\hline Number & 5 & 4 & 5 & 2 & 3 \\
\hline Sex, \% women & 100 & 100 & 100 & 100 & 100 \\
\hline Age, mean, y & 55 & 45 & 58 & 32 & 53 \\
\hline Race, \% white & 60 & 100 & 80 & 0 & 100 \\
\hline Certified (CMA or RMA), \% & 25 & 66 & 25 & 0 & 50 \\
\hline Mean years as MA & 13 & 13 & 29 & 1 & 16 \\
\hline Mean years at this office & 5 & 9 & 5 & 1 & 8 \\
\hline \multicolumn{6}{|l|}{ Offices } \\
\hline Ownership of practice & Physician & $\begin{array}{l}\text { Health } \\
\text { system }\end{array}$ & $\begin{array}{l}\text { Health } \\
\text { system }\end{array}$ & $\begin{array}{l}\text { Health } \\
\text { system }\end{array}$ & Physician \\
\hline \multicolumn{6}{|l|}{ Patient composition, \% } \\
\hline Medicare & 16 & 25 & 55 & 15 & 15 \\
\hline Medicaid/uninsured & 16 & 1 & 2 & 51 & 5 \\
\hline Private insurance & 68 & 74 & 43 & 34 & 80 \\
\hline Office location & $\begin{array}{c}\text { Rural/ } \\
\text { suburban }\end{array}$ & Urban & Suburban & Urban & $\begin{array}{c}\text { Rural/ } \\
\text { suburban }\end{array}$ \\
\hline
\end{tabular}

Table 3. Perceived Benefits of Nurses and MAs in Family Medicine Offices

\begin{tabular}{|c|c|c|}
\hline Group & Clinician Perceptions & MA Perceptions \\
\hline \multirow{7}{*}{$\begin{array}{l}\text { Nurses (RNs } \\
\text { and LPNs) }\end{array}$} & Starting IVs & Starting IVs \\
\hline & Ability to answer patient questions & \multirow{6}{*}{$\begin{array}{l}\text { RNs only (not LPNs): ability to } \\
\text { answer patient questions }\end{array}$} \\
\hline & Assessing and triaging patient clinical concerns & \\
\hline & Teaching patients & \\
\hline & $\begin{array}{l}\text { Ability to provide clinical information } \\
\text { (eg, results) to patients }\end{array}$ & \\
\hline & Independence to work with less supervision & \\
\hline & Less on-the-job training needed & \\
\hline \multirow[t]{5}{*}{ MAs } & Lower salary & Lower salary \\
\hline & Trained in clerical office work & Trained in clerical office work \\
\hline & Able to serve in back and front office & Able to serve in back and \\
\hline & More malleable to on-the-job training & front office \\
\hline & $\begin{array}{l}\text { Often from lower SES; may connect with } \\
\text { lower-SES patients }\end{array}$ & $\begin{array}{l}\text { Sufficient knowledge to handle } \\
\text { all office-based medical ques- } \\
\text { tions, education, and care }\end{array}$ \\
\hline
\end{tabular}

Table 3 illustrates clinicians and MAs' differing views on MA roles, which were often expressed by comparing MAs and nurses. This disagreement introduced a major constraint in the clinician-MA relationship. MAs stressed that most of their clinical work (checking in patients, assisting clinicians) was similar to office nurse tasks. Clinicians, however, expressed concern about MAs' clinical competence, especially unsupervised one-on-one patient interactions. One-half of the clinicians made statements similar to that of this physician: "They're not nurses; they don't have the training that nurses have. [Patients] will look to that MA for some clinical guidance, but they're not clinical." The MA viewpoint differed; as one commented, "In the family medicine office, I think the MAs do about everything the RNs [registered nurses] do now." Another noted, "To be honest with you, MAs do more than nurses do. They don't get paid as much, but they do more." MAs generally resented earning less for what they often perceived as nurse-level work, whereas clinicians indicated that MA pay is commensurate with their lower level of training and expertise. The MAs' association of their own work roles with those of nurses suggests they perceived additional freedoms in the relationship (eg, professional autonomy) that in varying degrees is restricted or constrained by the physicians' concerns about MAs' limited clinical expertise.

\section{Clinician-MA Relationships}

Tensions between MAs' and clinicians' perceptions of MA clinical capabilities resulted in a relational model we call trust and verify, characterized by different configurations of physician trust and verification of the MA's clinical activities. The varying approaches are informed by clini- 
cians' roles in MA hiring and management and with the degree of social familiarity between MAs and clinicians. The case studies presented in Table 1 (and further discussed below) depict how these factors help define team function and, as with all relationships in organizational contexts, demonstrate self-organizing and nonlinear patterns.

\section{Trust and Verification}

Trust and verification describes the range of MA clinical independence allowed by clinicians. In the interviews, clinicians discussed their role in determining how MAs interact clinically with patients, including giving education and advice, relaying test results, answering patient questions, and performing clinical procedures. Clinicians at physician-owned offices (offices 1 and 5) were most restrictive; as one stated, "You have to guide your MA as to what information to give out, what not to give out, what you want them to say and for them not to put their own spin on the information because it may not be accurate." Another physician owner controlled the information MAs gave with "a notebook that is sort of canned answers, so that I make sure that the MAs don't go over the top talking about things that they shouldn't talk about." Other clinicians came to trust and gave MAs greater clinical independence, however. This relationship was most evident in offices 2 and 3, which were mature, previously independent, but now health system-owned offices; as one clinician stated, "I would say I leave my MA pretty much on her own. I mean, we've worked with our staff for multiple years, and I guess I know what their deficiencies are." Physicians at the newest office, office 4 , had the least experience in supervising MAs, and felt the least empowered; one commented, "I have nothing in particular that I think is effective [to help me supervise MAs], and I sometimes fret over the communication train wreck that we function in."

In contrast, MAs in every office expressed confidence in their clinical skills. All MAs reported regularly answering patients' questions and giving advice, and a majority expressed confidence in their training, saying, for example, "we learned everything we need in school," and in their previous personal and work experiences. As one put it, "When I started here, I had a book of notes that had been passed on to me, ...but I feel like my clinical background allows me to adjust the instructions I'm giving to the patients, given my own personal life experiences also." An MA in office 4 described how she learned about medications from a licensed practical nurse at a previous position and could now regularly talk to patients about them: "They did teach us in school that we would be giving medicines, but never that we would be calling them in, explaining to patients what they are, what they are used for, teaching patients about the medications."

We did not necessarily find linear relationships between trust in an MA's clinical expertise and a clinician's actual verification of MA clinical activities. As illustrated in office 5, lack of trust led to more verification, while in office 4 , lack of trust existed without verification. A factor that helped explain clinicians' trust and verification was familiarity with the MAs, partially informed by the clinician's role in their hiring and daily management.

\section{Hiring and Management of MAs}

Hiring and managing MAs varied primarily between physician and health system ownership. Physician owners made hiring decisions (offices 1 and 5), but health system physicians did not (offices 2, 3, and 4). One health system physician stated, "I have no way of selecting the MA. I think there is some shaping done to the MA relationship, and you're either going to have a good fit or not." Physician owners were more knowledgeable about MA education and certification, and more aware of office training; one physician owner stated that "we pretty much just train the MAs when we hire them.... Most training is by other MAs here or by our office manager; she's an MA also." In fact, all the MAs received training from other MAs. All clinicians reported directing behavior by feedback, "[MAs] are willing to take criticism.... They try to better their work by taking the criticism to heart." None of the clinicians or MAs, however, described any structured evaluations or feedback from the clinicians to the MAs.

\section{Social Familiarity}

Social familiarity between MAs and clinicians was higher at the older offices with fewer turnovers. Working primarily with 1 physician also fostered familiarity. In every office, except office 2, there was a primary clinician-MA dyad. At office 2, all MAs worked with all clinicians, and familiarity was generated through years of working together and a congenial culture such as was observed during the staff meeting. We observed meetings at all offices except one. In offices 2,3 , and 5, socializing and sharing personal information was encouraged, and staff and clinicians ate lunch together. Clinicians offered supportive comments to MA suggestions and questions at these meetings. In office 3, the MA supervisor helped set the agenda. In the office 4 staff meeting, we observed unclear physician leadership and clinician-dominated conversations. At this office, only the MAs ate during the meeting. Unstructured socializing between clinicians and MAs during lunch hours or breaks was rarely observed at this office, in contrast to the other 4 offices. 


\section{Variations in Trust and Verify Model}

Table 4 shows variation in the components of the trust and verify model across the offices. Clinicians in offices 1 and 4 exhibited low trust and low verification of MA activities. In office 1 , experienced clinician owners used explicit protocols to guide MA clinical behavior and establish the tone of working relationships; however, although regular MA-clinician meetings were held and some feedback was exchanged, there was little direct oversight of MA activities, and MAs acknowledged bending or violating the protocols. Office 4 was noteworthy in that 3 new physicians in a system-owned practice took a hands-off approach to the MA relationship, making little effort to become familiar with MAs and providing little guidance or feedback. These clinicians' relative inexperience and lack of management skills along with their undefined roles in MA supervision within a larger health system produced relationships that, based on reported turnover, were short lived and had little resemblance to teams.

Office 5, a mature, 2-clinician physician-owned practice, had low clinician trust, but in contrast to offices 1 and 4 , had strong verification practices. MAs were hired and supervised by the physician owner and given clear guidelines regarding patient communications. Clinician monitoring was enhanced by a tight, centralized office space. MA-clinician socialization was frequent and turnover low, and although informal feedback channels were relatively open, there was little formal feedback.

Offices 2 and 3 demonstrated high clinician trust accompanied by low verification. Both had established physician-owner legacy structures with protocols and staff undergoing transitions to health system ownership. Office 2, a mature 3-clinician practice had recently transitioned to health system ownership and maintained many of its protocols and long-term staff. MA turnover was low, and clinicians trusted and considered MAs well trained based on prior formalized evaluations and years of experience working together. Clinicians reported little need or effort to verify MA activities. In office 3 , a younger clinician assumed

Table 4. Components of the Trust and Verify Model by Office

\begin{tabular}{llll}
\hline Office & Trust and Verification & $\begin{array}{l}\text { MA Hiring and } \\
\text { Management }\end{array}$ & $\begin{array}{l}\text { Social } \\
\text { Familiarity }\end{array}$ \\
\hline Office 1 & Low trust, low verification & Hands on & High \\
Office 2 & High trust, low verification & Hands on & High \\
Office 3 & High trust, low verification & Hands off & High \\
Office 4 & Low trust, low verification & Hands off & Low \\
Office 5 & Low trust, high verification & Hands on & High \\
\hline MA = medical assistant. & & \\
\hline
\end{tabular}

an established practice including MAs. MA-clinician familiarity was high, and regular formal and informal staff meetings provided opportunities for feedback; however, the new health system now hired and managed MAs, leaving the physician's ongoing role in MA supervision and evaluation unclear.

\section{DISCUSSION}

Medical assisting is one of the fastest-growing professions in America, and the field is expected to grow $31 \%$ by the end of $2020 .{ }^{19}$ Success in practice models such as the PCMH requires strong relationships between the professionals having main contact with patients and their colleagues, who, in these smaller offices, are clinicians (physicians and nurse practitioners) and MAs. ${ }^{13-15}$ We intensively studied 5 small family medicine offices to better understand their relationships. The MA roles and work tasks reported and observed here corroborate the shared belief that the MA's primary responsibility is to maintain patient flow. ${ }^{2,5,6}$ In more cognitive domains of clinical communications and advice giving, however, we found conflict between clinicians' and MAs' perceptions of roles. Clinicians differed in the strategies they used to monitor and direct MA activities. We applied the conceptual language of CRPR to depict how these conflicts were handled under different conditions of trust and verification.

As self-organizing systems directed toward common clinical work goals, MA-clinician teams adapted to changing conditions with varying degrees of freedom (autonomy, opportunities, openings) and constraints (checks, boundaries, limits) while using channels of feedback and response. ${ }^{14,15,20-22}$ For example, as MAs replaced nurses in family medicine settings, clinicians adapted to new system constraints by limiting certain kinds of MA-patient communications; however, the training of MAs by other MAs in these offices perpetuated patient flow as the primary clinical goal, and modeled and taught independent MA-patient communications. These cultural norms acted as a constraint to major change. This constraint may help explain the failure of some recent experiments to move MAs into new roles as health promoters. ${ }^{8,9}$ In a trial wherein MAs were trained as health coaches, the authors explained their poor results in part as follows: "MAs are trained to carry out concrete clinical tasks such as measuring blood pressures, conducting a focused patient history, and managing patient flow. The duties of MAs in their roles as health coaches...interfered with 
what they saw as their primary responsibilities-managing patient flow and conducting time-limited clinical tasks."23 Our study suggests the utility of CRPR to explain these clinical trial findings. It may be that before simply training an MA for a different task, more attention needs to be paid to the web of relationships within which the MAs work. ${ }^{13,14}$

Lack of trust constrained clinician-MA relationships in some offices, while presence of trust offered an important degree of freedom for others. Lanham and colleagues ${ }^{24}$ posit that low trust limits the ability to form relationships and leads to nonexistent teams, In the RCC practice adaptive reserve model of Miller et $\mathrm{al}_{1}{ }^{14}$ establishing trust is central. Although none of the offices we studied had NCQA PCMH certification at the time of the study, follow-up revealed that the 2 offices where trust was high (offices 2 and 3 ) have since received this certification. The office with no functioning team underwent considerable changes in personnel and structure, established new relationships, and did achieve PCMH certification. Although this study is insufficient to demonstrate causation, these cases add to the findings from structured transformation projects such as the National Demonstration Project that trust within RCC is key when assessing PCMH potential. ${ }^{14}$

There are limitations to this study. As a qualitative study, the goal was not to be generalizable to all small offices, but to explore the clinician-MA relationship within the model of RCC. The offices were from a single geographic region. Although they were purposefully sampled for varied characteristics, offices in other geographic areas may differ. As this was a preliminary study, we were unable to confirm findings in additional offices, which would have increased the strength of transferability. Strategies to increase our reliability included studying 5 offices with varied practice characteristics and using data analysts from different backgrounds (family medicine, anthropology, psychology, and sociology). As none of the offices had PCMH certification during the study, clinician-MA relationships within established $\mathrm{PCMHs}$ is an area for further research.

MAs have become an integral part of many family medicine offices, yet their relationships to the clinicians with whom they work are understudied. , $^{2,6,7}$ This lack of understanding may help explain why, despite decades of attempts to develop them, "primary care teams have not become the dominant paradigm." 25 The current changes in family medicine and successful practice development require optimizing the clinicianMA relationship. CRPR provides a theoretical framework wherein our findings begin to define some of the constraints and freedoms in patterns of relating in small family medicine offices. We describe an RCC model, trust and verify, to describe clinician-MA relationships. Crabtree and colleagues ${ }^{10}$ posit that practice development will require physicians to move to a model of "heedful interrelating," wherein physicians are aware of the effect their actions have on staff. Our findings may assist small offices undergoing practice transformation and guide future research to better understand how best to partner with MAs in the family medicine setting.

To read or post commentaries in response to this article, see it online at http://www.annfammed.org/content/12/2/150.

Key words: Allied health personnel; work relationships; interpersonal relations; medical staff; practice dynamics; teams; primary care; practice-based research

Submitted September 17, 2012; submitted, revised, July 11, 2013; accepted August 2, 2013.

Funding support: The study was supported by the Agency for Healthcare Research and Quality (AHRQ) through grant 1R03HS01903001 and also by the National Center for Research Resources and the National Center for Advancing Translational Sciences, National Institutes of Health (NIH), through grant 8 UL1 TR000077-04.

Disclaimer: The content of this article is solely the responsibility of the authors and does not necessarily represent the official views of AHRQ or of the NIH.

\section{References}

1. Aita V, Dodendorf DM, Lebsack JA, Tallia AF, Crabtree BF. Patient care staffing patterns and roles in community-based family practices. J Fam Pract. 2001;50(10):889.

2. Taché S, Chapman S. The expanding roles and occupational characteristics of medical assistants: overview of an emerging field in allied health. J Allied Health. 2006;35(4):233-237.

3. Taché S, Chapman S. What a medical assistant can do for your practice. Fam Pract Manag. 2005;12(4):51-54.

4. Examples of Medical Assistant Scope of Practice Rules by State. http://www.medicalassistant.net/scope_of_practice_by_state.htm. Accessed May 7, 2012.

5. Chapman S, Chan M, Bates T. Medical Assistants in Community Clinics: Perspectives on Innovation in Role Development. San Francisco, CA: Center for the Health Professions, University of California San Francisco; 2010.

6. Chapman S, Marks A, Chan M. Research Brief: The Increasing Role of Medical Assistants in Small Primary Care Physician Practice: Key Issues and Policy Implications. San Francisco, CA: Center for the Health Professions, University of California San Francisco; 2010.

7. Taché $S$, Hill-Sakurai L. Medical assistants: the invisible "glue" of primary health care practices in the United States? J Health Organ Manag. 2010;24(3):288-305.

8. Ferrer RL, Mody-Bailey P, Jaén CR, Gott S, Araujo S. A medical assistant-based program to promote healthy behaviors in primary care. Ann Fam Med. 2009;7(6):504-512.

9. Bodenheimer T, Laing BY. The teamlet model of primary care. Ann Fam Med. 2007;5(5):457-461.

10. Crabtree BF, McDaniel RR, Nutting PA, Lanham HJ, Looney AJ, Miller WL. Closing the physician-staff divide: a step toward creating the medical home. Fam Pract Manag. 2008;15(4):20-24. 
11. Moore LG. Creating a high-performing clinical team. Fam Pract Manag. 2006;13(3):38-40.

12. Chesluk BJ, Holmboe ES. How teams work-or don't-in primary care: a field study on internal medicine practices. Health Aff (Millwood). 2010;29(5):874-879.

13. Safran DG, Miller W, Beckman H. Organizational dimensions of relationship-centered care. Theory, evidence, and practice. J Gen Intern Med. 2006;21(Suppl 1):S9-S15.

14. Miller WL, Crabtree BF, Nutting PA, Stange KC, Jaen CR. Primary care practice development: a relationship-centered approach. Ann Fam Med. 2010;8(Suppl 1):S68-S79; S92.

15. Suchman AL. A new theoretical foundation for relationship-centered care. Complex responsive processes of relating. J Gen Intern Med. 2006;21(Suppl 1):S40-S44.

16. Crabtree B, Miller W. Doing Qualitative Research. 2nd ed. Thousand Oaks, CA: Sage Publications; 1999.

17. Miller WL, Crabtree BF. Qualitative analysis: how to begin making sense. Fam Pract Res J. 1994;14(3):289-297.

18. Yin R. Case Study Research Design and Methods. 3rd ed. Bickman L, Rog D, eds. Thousand Oaks, CA: Sage Publications; 2003.
19. Lockard C, Wolf M. Occupational employment projections to 2020. Mon Labor Rev. 2012;135(1):85-108.

20. Miller WL, McDaniel RR Jr, Crabtree BF, Stange KC. Practice jazz: understanding variation in family practices using complexity science. J Fam Pract. 2001;50(10):872-878.

21. Crabtree BF, Miller WL, Stange KC. Understanding practice from the ground up. J Fam Pract. 2001;50(10):881-887.

22. Stroebel CK, McDaniel RR Jr, Crabtree BF, Miller WL, Nutting PA, Stange KC. How complexity science can inform a reflective process for improvement in primary care practices. Jt Comm J Qual Patient Saf. 2005;31(8):438-446.

23. Saba GW, Taché S, Ward L, Chen EH, Hammer H. Building teams in primary care: what do nonlicensed allied health workers want? Perm J. 2011;15(3):9-17.

24. Lanham HJ, McDaniel RR Jr, Crabtree BF, et al. How improving practice relationships among clinicians and nonclinicians can improve quality in primary care. Jt Comm J Qual Patient Saf. 2009; 35(9):457-466.

25. Grumbach K, Bodenheimer T. Can health care teams improve primary care practice? JAMA. 2004;291(10):1246-1251. 